\title{
Addressing Lifestyle Issues: Thoughts on Adopting a Systems Approach
}

\author{
Terrence Thomas* \\ Department of Agribusiness, North Carolina A\&T State University, USA
}

*Corresponding author: Terrence Thomas, Department of Agribusiness, Applied Economics and Agri science Education, College of Agriculture and Environmental Sciences, North Carolina A\&T State University, Greensboro, North Carolina, USA

To Cite This Article: Terrence Thomas. Addressing Lifestyle Issues: Thoughts on Adopting a Systems Approach. Am J Biomed Sci \& Res. 2019 - 5(5). AJBSR.MS.ID.000941. DOI: 10.34297/AJBSR.2019.05.000943.

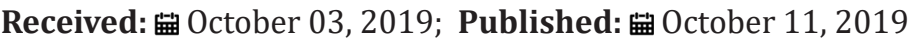

\section{Opinion}

Lifestyle is a social phenomenon. It is the interests, way of thinking, way of living, or orientation to life of an individual, group, community or culture. Lifestyle results from several interacting factors that produce a predisposition to act in certain ways or a certain style of behaving toward one's environment, broadly speaking. One useful way to look at lifestyle is to consider lifestyle as a mediating variable between values and observed behavior. In other words, lifestyle is a way of expressing one's values toward a thing or phenomenon [1]. Take for example health, a healthy life style emanates from the value one places on eating a balanced diet, shopping for the ingredients that make up this balanced diet, regular exercise, avoiding harmful habits, seeking out useful information on achieving and maintaining a healthy lifestyle and the value placed on the opinion of significant others that may influence one's lifestyle choices among many other things. So then, lifestyle as it relates to health is a complex concept; complex in the sense that its expression is the result of several interacting components. Complexity is a primary feature of social phenomena and lifestyle issues whether they relate to health, family or the environment are good everyday examples. Complexity is a staple distinguishing feature of a system, which can be understood along several dimensions.

From a practical problem-solving stance, we are interested in two features of complexity: inherent complexity and dynamic complexity. In the case of inherent complexity, the system has a great many interacting parts with several possible ways of interacting and feedback loops. Inherent complexity is important because it endows a system with the special quality of emergence -a case where certain properties of the system emerge as a result of the complex nature of the system. In instances of dynamic complexity, one's action on the system to solve a problem has a different outcome over time, that is, the impact over the short run is different from that observed over the long run.

These two features of a complex system have implications for our problem-solving approach in tackling lifestyle issues and other social problems, where complexity is the order of the day. In systems that are inherently complex and dynamic much time is needed to develop a reasonably good understanding of the system. In these systems, given the feature of emergence-new properties of the system emerge as a result of their inherent complexity; and the dynamic nature of the system's interaction with the environment, it is not likely that one can generate the amount of data that will produce the level of understanding needed to craft the single right solution. In such instances, the classic linear approach to problem solving -define the problem, identify and assess alternative solutions and "select one, the best one"-is not suited for complex problem situations. Additionally, focusing on a single variable in a problem situation defined by multiple interacting variables is not likely to produce the desired impact. In general, problems are of two types: puzzles and mysteries [2]. In the case of puzzles, if one has the right amount of data, solving the problem is routine; for example, predicting the development of a storm and tracking its movement. On the other hand, in the case of a mystery no amount of data disclosure will lead to the formulation of a definitive solution -the single right solution. Thus, finding solutions to mysteries is a process of iterating our way to a progressively better solution over time. For instance, no amount of data will reveal the insights that will make possible the formulation of a single right solution for building a stable and prosperous Afghanistan or Haiti or improving the quality of life for underserved residents in the food desert of Eastern Greensboro, North Carolina, US. The inherent, dynamic and complex nature of mysteries make them unresponsive to applying a linear search for a single best solution. Instead, a systems approach 
that emphasizes learning in order to develop a deep understanding of the problem situation, with focus on a multiple variable approach, as one iterates his/her way to a progressively better solution is the viable alternative.

Attempts to address problems linked to lifestyle issues usually start with identifying and working on a single variable at a time. For example, in addressing the food desert problem, the most popular approach is to improve access to affordable healthy food. Usually, a coordinated, simultaneous attention is not given to other variables operating in the problem situation. In addressing lifestyle issues, more attention should be paid to the individuals who are the objects and agents of the sought-after solution. Individuals are the agents of the solution because they must buy into and make the solution an integral part of their lived experience for it to work. Therefore a deep understanding of the individuals and their place in the system in which they exist is central to developing real innovative solutions that matter. The popular quantitative analytical approach rooted in rational scientific analysis that relies on numbers is the staple of problem-solving methods. But in increasingly complex problem-solving situations where people are the focus of attention, the rational analytic approach that depends on numbers seem inadequate. Although numbers can tell a part of the story, they are not good for telling the story behind the numbers, providing insight into the fears and aspirations, the hidden motives behind actions, the rich story of the daily lives of people and uncovering unarticulated needs and desires of people. One can only get access to this rich data through direct observation employing ethnographic methods. These methods rest on the philosophy Aristotle espoused--a second road to truth is rhetorical argument based on conversation, the first being the rational scientific analysis that depends on numbers. So then, solving a lifestyle related problem always involve changing the situation represented by a complex of factors where people are at the center of this complex.

Social problems that are worth solving are mysteries; it is not likely that success can be realized solving our way to a single best solution and applying a linear approach. To improve the likelihood of success in complex problem situations, it will be necessary to iterate our way to progressively better solutions, to do this we must embrace complexity and systems thinking.

This means developing an approach to problem solving that accepts as central to the problem solving process the following actions: develop deep empathy for the people involved and the problem situation, capture the lived experience of the people in the problem situation, assess resources in the problem situation that can be leveraged to support the problem solving process, anticipate, perceive and envision scenarios, create personas to model and observe behavior under different scenarios, and design solutions that fit harmoniously with the social and emotional complex that define the reality of the people being served. Acquiring a deep understanding of the lived experience and the existing situation of the people served and involving them in co-creating the solution will likely increase their interest in and the personal significance of the solution.

The forgoing discussion is not suggesting discarding the quantitative and or linear approach to problems solving. Rather we should seek to adopt a systems approach as a tool to augment our problem-solving repertoire. To make progress we don't have to throw the baby away with the bath water, we just need to refresh the water

\section{References}

1. Karen Brunsø, J Victor Scholderer, Klaus G Grunert(2004) Testing relationships between values and food- related lifestyle: results from two European countries. Appetite 43(2): 195-205.

2. Jeanne Liedtka, Tim Oglivie(2011) Design for Growth, Columbia University Press, USA. 